\title{
Cryptogenic stroke
}

\section{Contemporary trends, treatments, and outcomes in the United States}

\author{
Shyam Prabhakaran, MD, MS, Steven R. Messé, MD, Dawn Kleindorfer, MD, Eric E. Smith, MD, MPH, \\ Gregg C. Fonarow, MD, Haolin Xu, MS, Xin Zhao, PhD, Barbara Lytle, PhD, Joaquin Cigarroa, MD, \\ and Lee H. Schwamm, MD
}

\author{
Correspondence \\ Dr. Prabhakaran \\ shyam1@uchicago.edu
}

Neurology: Clinical Practice October 2020 vol. 10 no. 5 396-405 doi:10.1212/CPJ.0000000000000736

\section{Abstract \\ Background}

Nationwide data on patients with cryptogenic stroke (CS) are lacking. We evaluated patient and hospital characteristics, in-hospital treatments, and discharge outcomes among patients with CS compared with other subtypes in the Get With The Guidelines (GWTG)Stroke registry.

\section{Methods}

We identified patients with ischemic stroke (IS) admitted to GWTGStroke participating hospitals between January 1, 2016, and September 30, 2017, with documented National Institutes of Health Stroke Scale (NIHSS) scale and stroke etiology (cardioembolic [CE], large artery atherosclerosis [LAA], small vessel occlusion [SVO], other determined etiology [OTH], or CS). Using multivariable logistic regression, we compared hospital treatments and discharge outcomes by subtype, adjusted for patient and hospital characteristics.

\section{Results}

Among 316,623 patients from 1,687 hospitals, there were 63,301 (20.0\%) patients with CS. In multivariable analysis, patients with CS received IV thrombolysis more often than other subtypes and had lower mortality than CE, LAA, and OTH but higher mortality than SVO. They were more likely to be discharged home than all other subtypes and be independent at discharge than LAA, OTH, or SVO.

\section{Conclusions}

In a large contemporary nationwide registry, CS accounted for $20 \%$ of ISs among patients with a documented stroke etiology. Patients with CS had a distinct profile of treatments and outcomes relative to other subtypes. Improved subtype documentation and further research into CS are warranted to improve care and outcomes for patients with stroke.

Ischemic stroke (IS) is a leading cause of disability in the United States. ${ }^{1}$ Outcomes and secondary prevention strategies vary by IS subtype or etiology; for example, anticoagulation is reserved primarily for cardioembolic (CE) subtype. In addition, IS subtype has research implications. There are several ongoing and recently completed trials for cryptogenic stroke (CS), as classified by the Trial of ORG 10172 in Acute Stroke Treatment (TOAST) criteria $^{2}$ that encompasses patients with an in-depth evaluation in whom no cause was identified or in

University of Chicago (SP), Pritzker School of Medicine; University of Pennsylvania (SRM); University of Cincinnati (DK); Hotchkiss Brain Institute (EES), University of Calgary, Calgary, Alberta, Canada; Ahmanson Cardiomyopathy Center (GCF), UCLA, Los Angeles, CA; Duke Clinical Research Institute (HX, XZ, BL), Durham, NC; Knight Cardiovascular Institute (JC), Oregon Health and Sciences University; and Department of Neurology (LHS), Massachusetts General Hospital.

Funding information and disclosures are provided at the end of the article. Full disclosure form information provided by the authors is available with the full text of this article at Neurology.org/cp. 
whom multiple potential causes were identified. For many patients, multiple specific phenotypes exist that may respond to targeted approaches including anticoagulation ${ }^{3}$ or patent foramen ovale (PFO) closure. ${ }^{4-7}$ Previous population-based and hospital-based cohorts have found that $\mathrm{CS}^{8-17}$ accounts for approximately $25 \%-40 \%$ of IS. However, there are relatively few data from the United States, mostly from geographically constrained population studies. ${ }^{12,15,17} \mathrm{We}$ therefore sought to evaluate patient and hospital characteristics, in-hospital treatments, and discharge outcomes among patients with CS compared with other subtypes in the Get With The Guidelines (GWTG)-Stroke registry, a large database of acute stroke discharges at over 2,000 hospitals in the United States, accounting for $\sim 60 \%$ of incident stroke cases.

\section{Methods}

\section{Data source}

The GWTG-Stroke registry is a quality improvement program for stroke care in the United States that promotes consistent adherence to the latest scientific management guidelines. Hospitals enter clinical and outcomes data via a web-based Patient Management Tool (PMT; IQVIA, Cambridge, MA). ${ }^{18}$

\section{Standard protocol approvals, registrations, and patient consents}

Data analysis was performed by the Duke Clinical Research Institute with local institutional review board approval. Under the common rule, sites are granted a waiver of informed consent, as the data are collected from medical records for use locally for quality improvement.

\section{Data collection and study population}

Using the GWTG-Stroke registry, we identified patients with final diagnosis of IS at participating hospitals between January 1, 2016, and September 30, 2017, with documented stroke etiology. The documentation of stroke etiology data collection questions and accompanying coding instructions were developed and finalized in 2015 and implemented in January 2016.

\section{Primary variable of interest}

CS subtype was the primary variable of interest and defined as occurring when physician documentation stated that a potential cause of stroke was not identified, or multiple possible causes were identified, following thorough diagnostic evaluation. Coding instructions noted that low- and moderate-risk potential CE sources such as PFO, heart failure with preserved ejection fraction, mitral annulus calcification, or atrial or ventricular arrhythmias other than atrial fibrillation or flutter (AF) were of uncertain significance and not adequate to ascribe stroke mechanism. The GWTGStroke coding instructions further subcategorized CS as stroke of (1) undetermined (when a potential etiology was
Patients with cryptogenic stroke were more likely to have better outcomes than all subtypes except those with small vessel disease.

not identified or documented following diagnostic evaluation), (2) unspecified (when there was no documentation of the results of the diagnostic evaluation), or (3) multiple competing etiologies (when a single etiology was uncertain between 2 or more possible causes following diagnostic evaluation). Other stroke subtypes, categorized according to the TOAST criteria, ${ }^{2}$ included CE, large artery atherosclerosis (LAA), small vessel occlusion (SVO), and other determined etiology (OTH).

\section{Patient and hospital characteristics}

Demographic data included age, sex, race/ethnicity, health insurance status, and arrival and admission data including onoff hours admission, mode of arrival such as emergency medical services (EMSs), walk-in, or transfer, National Institutes of Health Stroke Scale (NIHSS) score (if documented), and times for arrival and thrombolytic drug administration. Medical history included the presence of AF, coronary artery disease (CAD), previous myocardial infarction (MI), carotid stenosis, diabetes mellitus, heart failure, hypertension, prosthetic heart valve, peripheral vascular disease (PVD), smoking status, previous stroke/TIA, ambulatory status before admission, and previous medications. Hospital characteristics including geographic region, the number of beds, hospital location, teaching and primary stroke center status, and annual volume of stroke by each type were also analyzed. In-hospital treatment included antithrombotic therapy and other risk factor-modifying medications at discharge.

\section{Outcomes}

Quality and achievement measures included thrombolytic therapy with IV tissue plasminogen activator (tPA) within 3 hours or 4.5 hours of last known well in eligible patients, administration of tPA within 1 hour of hospital arrival, early antithrombotic therapy, anticoagulation for AF, deep venous thrombosis prophylaxis, lipid lowering, statin initiation, and low-density lipoprotein measurement, smoking cessation, dysphagia screening, stroke education, consideration for rehabilitation, and defect-free care. Ambulatory status, mortality, modified Rankin Scale (mRS) score, discharge destination, and length of stay (LOS) were also analyzed at the time of hospital discharge as outcomes. Because stroke severity is an important predictor of these outcomes, we included only the subset of patients with documented NIHSS score for these analyses $(\mathrm{n}=316,263)$. 


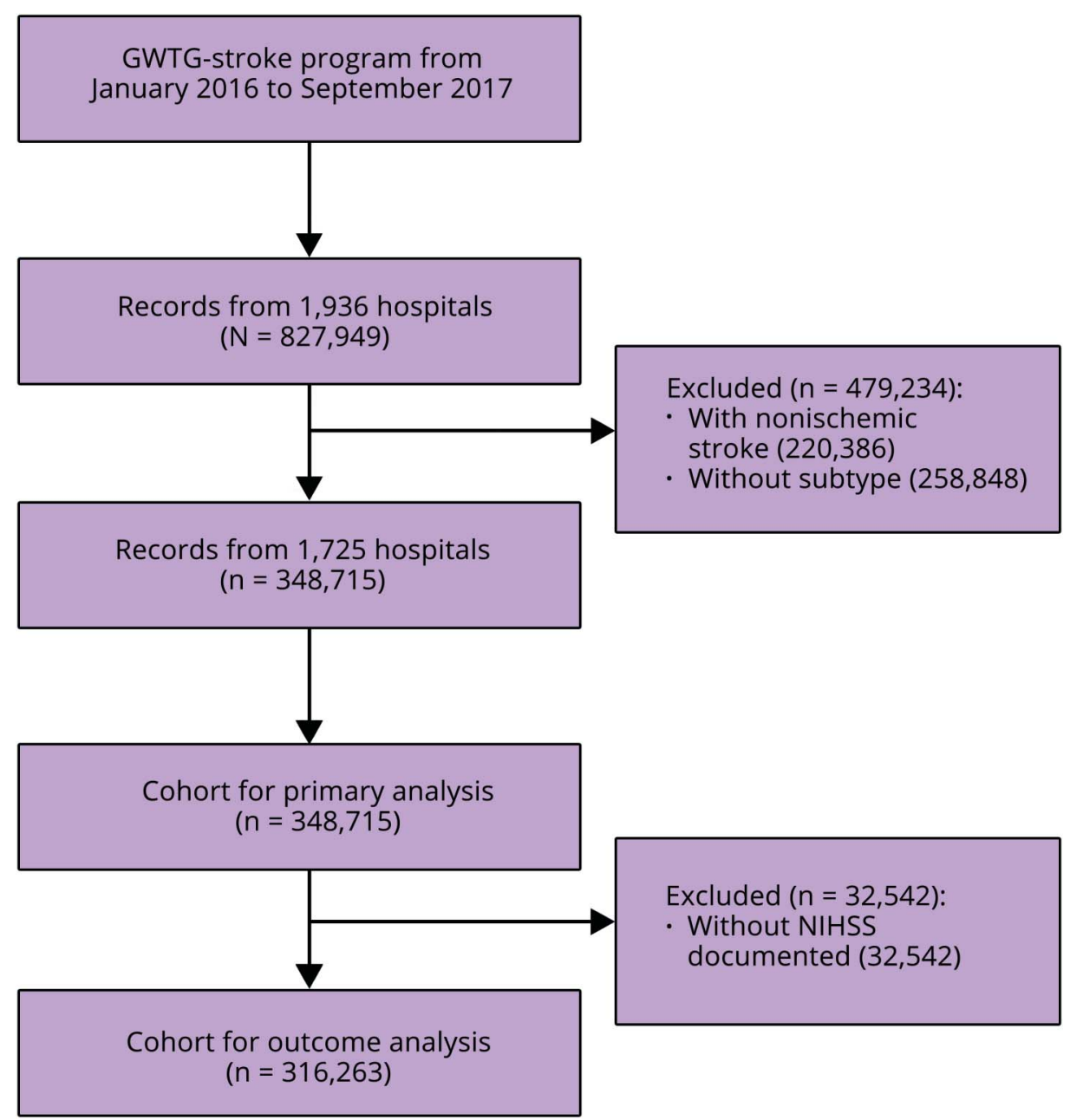

\section{Statistical Analysis}

All statistical analyses were performed using SAS Version 9.3 software (SAS Institute, Cary, NC). Baseline patient and hospital characteristics were described overall and compared across the 5 stroke subtypes. Categorical variables were presented as counts and percentages, and the difference between groups was tested using Pearson $\chi^{2}$ tests. Continuous variables were presented as mean and SDs or medians with 25 th and 75 th percentiles, and the difference between groups was tested using the KruskalWallis test.

To further evaluate the association between different documented stroke etiology categories with outcomes, multivariable logistic regression models were performed in those with documented NIHSS scores. Both unadjusted and adjusted odds ratios and 95\% confidence intervals are reported. The generalized estimating equation method with exchangeable working correlation matrix was applied to provide valid inference after accounting for the within-site correlation. Covariates in the model included age, sex, race, relevant vascular risk factor medical history elements (atrial fibrillation, prosthetic heart valve, previous stroke or TIA, CAD or previous MI, carotid stenosis, PVD, hypertension, dyslipidemia, and smoking), arrival during off hours (vs regular hours), EMS arrival, initial NIHSS score, annual IS admission, bed size, region, hospital type (academic vs nonacademic), and urban/rural location. In this cohort, covariates had very low missing rates. Missing sex $(0.02 \%)$ and hospital characteristics $(0.01 \%-1.03 \%$ missing $)$ were excluded, whereas other missing variables were imputed as follows: race (0.02\% missing) imputed to "white," mode of arrival (2.4\% missing) imputed to "No EMS," and medical history panel (missing $0.03 \%$ ) imputed to "No." Although insurance status was missing $15.2 \%$, it was not included in multivariable models.

In an identical manner and to evaluate our findings in patients with CS of undetermined etiology, sensitivity analyses were performed after excluding patients with CS with unspecified or multiple etiologies and patients with CS 
Table 1 Baseline and discharge characteristics in the primary analysis cohort with documented IS subtype $(n=348,715)$

\begin{tabular}{|c|c|c|c|c|c|c|c|c|c|c|c|c|c|}
\hline \multirow{2}{*}{$\begin{array}{l}\text { Variable } \\
\text { Demographics }\end{array}$} & \multicolumn{2}{|c|}{$\begin{array}{l}\text { Overall } \\
(n=348,715)\end{array}$} & \multicolumn{2}{|c|}{$\begin{array}{l}\text { CS } \\
(n=69,857)\end{array}$} & \multicolumn{2}{|c|}{$\begin{array}{l}\text { LAA } \\
(n=81,394)\end{array}$} & \multicolumn{2}{|c|}{$\begin{array}{l}\text { CE } \\
(n=96,592)\end{array}$} & \multicolumn{2}{|c|}{$\begin{array}{l}\text { SVO } \\
(n=82,542)\end{array}$} & \multicolumn{2}{|c|}{$\begin{array}{l}\text { Stroke of } \\
\text { other } \\
\text { determined } \\
\text { etiology } \\
(n=18,330)\end{array}$} & \multirow[t]{2}{*}{$p$} \\
\hline & & & & & & & & & & & & & \\
\hline \multicolumn{14}{|l|}{ Age in y } \\
\hline Mean & 70.12 & & 68.35 & & 69.89 & & 73.96 & & 69.29 & & 61.32 & & $<0.0001$ \\
\hline SD & 14.44 & & 14.85 & & 13.70 & & 13.87 & & 13.55 & & 16.86 & & \\
\hline \multicolumn{14}{|l|}{ Sex } \\
\hline Male & 176,286 & 50.55 & 34,924 & 49.99 & 43,339 & 53.25 & 46,459 & 48.10 & 42,172 & 51.09 & 9,392 & 51.24 & $<0.0001$ \\
\hline Missing & 91 & 0.03 & 9 & 0.01 & 16 & 0.02 & 23 & 0.02 & 36 & 0.04 & 7 & 0.04 & \\
\hline \multicolumn{14}{|l|}{ Race } \\
\hline White & 237,984 & 68.25 & 47,691 & 68.27 & 55,866 & 68.64 & 70,335 & 72.82 & 52,367 & 63.44 & 11,725 & 63.97 & $<0.0001$ \\
\hline Black & 59,974 & 17.20 & 12,884 & 18.44 & 13,443 & 16.52 & 13,110 & 13.57 & 17,072 & 20.68 & 3,465 & 18.90 & \\
\hline Hispanic & 24,395 & 7.00 & 4,693 & 6.72 & 5,834 & 7.17 & 6,018 & 6.23 & 6,356 & 7.70 & 1,494 & 8.15 & \\
\hline Asian & 10,776 & 3.09 & 1,752 & 2.51 & 2,504 & 3.08 & 2,854 & 2.95 & 3,033 & 3.67 & 633 & 3.45 & \\
\hline Native American & 1,301 & 0.37 & 315 & 0.45 & 286 & 0.35 & 313 & 0.32 & 290 & 0.35 & 97 & 0.53 & \\
\hline $\begin{array}{l}\text { Native Hawaiian or Pacific } \\
\text { Islander }\end{array}$ & 1,102 & 0.32 & 159 & 0.23 & 245 & 0.30 & 318 & 0.33 & 294 & 0.36 & 86 & 0.47 & \\
\hline UTD & 13,099 & 3.76 & 2,355 & 3.37 & 3,203 & 3.94 & 3,626 & 3.75 & 3,095 & 3.75 & 820 & 4.47 & \\
\hline Missing & 84 & 0.02 & 8 & 0.01 & 13 & 0.02 & 18 & 0.02 & 35 & 0.04 & 10 & 0.05 & \\
\hline \multicolumn{14}{|l|}{ Insurance Status } \\
\hline Private & 133,054 & 38.16 & 29,696 & 42.51 & 29,903 & 36.74 & 36,447 & 37.73 & 29,203 & 35.38 & 7,805 & 42.58 & $<0.0001$ \\
\hline Medicare & 110,996 & 31.83 & 20,727 & 29.67 & 25,860 & 31.77 & 3,4883 & 36.11 & 25,535 & 30.94 & 3,991 & 21.77 & \\
\hline Medicaid & 36,009 & 10.33 & 8,093 & 11.59 & 8,651 & 10.63 & 8,261 & 8.55 & 8,812 & 10.68 & 2,192 & 11.96 & \\
\hline Self-pay or None & 13,179 & 3.78 & 2,721 & 3.90 & 3,332 & 4.09 & 2,549 & 2.64 & 3,632 & 4.40 & 945 & 5.16 & \\
\hline Not Documented & 1,416 & 0.41 & 310 & 0.44 & 291 & 0.36 & 303 & 0.31 & 399 & 0.48 & 113 & 0.62 & \\
\hline Missing & 54,061 & 15.50 & 8,310 & 11.90 & 13,357 & 16.41 & 14,149 & 14.65 & 14,961 & 18.13 & 3284 & 17.92 & \\
\hline
\end{tabular}

\section{Arrival and Admission data}

\begin{tabular}{|c|c|c|c|c|c|c|c|c|c|c|c|c|c|}
\hline \multicolumn{14}{|l|}{ Stroke onset location } \\
\hline Not in a health care setting & 310,707 & 89.10 & 62,858 & 89.98 & 72,677 & 89.29 & 83,342 & 86.28 & 75,768 & 91.79 & 16,062 & 87.63 & $<0.0001$ \\
\hline Chronic health care facility & 16,448 & 4.72 & 3,122 & 4.47 & 3,723 & 4.57 & 5,875 & 6.08 & 3,101 & 3.76 & 627 & 3.42 & \\
\hline $\begin{array}{l}\text { Stroke occurred while the } \\
\text { patient was an inpatient in } \\
\text { your hospital }\end{array}$ & 9,271 & 2.66 & 1,662 & 2.38 & 1,957 & 2.40 & 3,592 & 3.72 & 1,359 & 1.65 & 701 & 3.82 & \\
\hline Outpatient health care setting & 4,290 & 1.23 & 916 & 1.31 & 941 & 1.16 & 1,094 & 1.13 & 1,057 & 1.28 & 282 & 1.54 & \\
\hline Another acute care facility & 5,803 & 1.66 & 901 & 1.29 & 1,609 & 1.98 & 2,014 & 2.09 & 855 & 1.04 & 424 & 2.31 & \\
\hline $\begin{array}{l}\text { Not documented or cannot be } \\
\text { determined }\end{array}$ & 1,904 & 0.55 & 374 & 0.54 & 401 & 0.49 & 583 & 0.60 & 329 & 0.40 & 217 & 1.18 & \\
\hline Missing & 292 & 0.08 & 24 & 0.03 & 86 & 0.11 & 92 & 0.10 & 73 & 0.09 & 17 & 0.09 & \\
\hline \multicolumn{14}{|l|}{ Arrival mode } \\
\hline EMS from home/scene & 157,712 & 45.23 & 30,616 & 43.83 & 37,664 & 46.27 & 48,073 & 49.77 & 34,465 & 41.75 & 6,894 & 37.61 & $<0.0001$ \\
\hline
\end{tabular}


Table 1 Baseline and discharge characteristics in the primary analysis cohort with documented IS subtype $(n=348,715)$ (continued)

\begin{tabular}{|c|c|c|c|c|c|c|c|c|c|c|c|c|c|}
\hline \multirow{2}{*}{$\begin{array}{l}\text { Variable } \\
\begin{array}{l}\text { Private transport/taxi/other } \\
\text { from home/scene }\end{array}\end{array}$} & \multicolumn{2}{|c|}{$\begin{array}{l}\text { Overall } \\
(n=348,715)\end{array}$} & \multicolumn{2}{|c|}{$\begin{array}{l}\text { CS } \\
(n=69,857)\end{array}$} & \multicolumn{2}{|c|}{$\begin{array}{l}\text { LAA } \\
(\mathrm{n}=81,394)\end{array}$} & \multicolumn{2}{|c|}{$\begin{array}{l}\text { CE } \\
(n=96,592)\end{array}$} & \multicolumn{2}{|c|}{$\begin{array}{l}\text { SVO } \\
(\mathrm{n}=82,542)\end{array}$} & \multicolumn{2}{|c|}{$\begin{array}{l}\text { Stroke of } \\
\text { other } \\
\text { determined } \\
\text { etiology } \\
(n=18,330)\end{array}$} & \multirow[t]{2}{*}{$p$} \\
\hline & 114,166 & 32.74 & 24,536 & 35.12 & 23,497 & 28.87 & 23,680 & 24.52 & 36,093 & 43.73 & 6,360 & 34.70 & \\
\hline Transfer from other hospital & 64,916 & 18.62 & 12,528 & 17.93 & 17,614 & 21.64 & 20,642 & 21.37 & 9,911 & 12.01 & 4,221 & 23.03 & \\
\hline ND or unknown & 2,213 & 0.63 & 438 & 0.63 & 537 & 0.66 & 490 & 0.51 & 609 & 0.74 & 139 & 0.76 & \\
\hline Missing & 9,708 & 2.78 & 1,739 & 2.49 & 2,082 & 2.56 & 3,707 & 3.84 & 1,464 & 1.77 & 716 & 3.91 & \\
\hline Arrival during off hours & 164,302 & 47.12 & 32,622 & 46.70 & 38,803 & 47.67 & 46,786 & 48.44 & 37,299 & 45.19 & 8,792 & 47.97 & $<0.0001$ \\
\hline NIH Stroke Scale (median) & 316,263 & 4.00 & 63,301 & 3.00 & 74,578 & 5.00 & 88,179 & 5.00 & 73,875 & 3.00 & 16,330 & 3.00 & $<0.0001$ \\
\hline 25th & & 1.00 & & 1.00 & & 2.00 & & 2.00 & & 1.00 & & 1.00 & \\
\hline 75th & & 9.00 & & 8.00 & & 12.00 & & 13.00 & & 5.00 & & 8.00 & \\
\hline Missing (\%) & & 9.31 & & 9.38 & & 8.37 & & 8.71 & & 10.50 & & 10.91 & \\
\hline \multicolumn{14}{|l|}{ Medical history } \\
\hline Hypertension & 265,847 & 76.29 & 52424 & 75.08 & 62,366 & 76.71 & 74,579 & 77.26 & 64,635 & 78.36 & 11,843 & 64.63 & $<0.0001$ \\
\hline Diabetes mellitus & 122,096 & 35.04 & 24,485 & 35.07 & 28,815 & 35.44 & 30,164 & 31.25 & 33,262 & 40.33 & 5,370 & 29.31 & $<0.0001$ \\
\hline Dyslipidemia & 164,451 & 47.19 & 33,611 & 48.14 & 38,232 & 47.02 & 46,787 & 48.47 & 38,802 & 47.04 & 7,019 & 38.31 & $<0.0001$ \\
\hline Previous stroke/TIA & 106,524 & 30.57 & 22,028 & 31.55 & 24,900 & 30.63 & 29,428 & 30.49 & 24,957 & 30.26 & 5,211 & 28.44 & $<0.0001$ \\
\hline Atrial fibrillation/flutter & 71,687 & 20.57 & 8,125 & 11.64 & 11,207 & 13.78 & 43,363 & 44.92 & 7596 & 9.21 & 1,396 & 7.62 & $<0.0001$ \\
\hline CAD/previous MI & 81,911 & 23.51 & 15,579 & 22.31 & 19,726 & 24.26 & 26,955 & 27.93 & 16,692 & 20.24 & 2,959 & 16.15 & $<0.0001$ \\
\hline PVD & 15,919 & 4.57 & 3,164 & 4.53 & 4230 & 5.20 & 4,642 & 4.81 & 31,73 & 3.85 & 710 & 3.87 & $<0.0001$ \\
\hline CHF & 34,645 & 9.94 & 5,894 & 8.44 & 6,809 & 8.37 & 15,079 & 15.62 & 5,709 & 6.92 & 1154 & 6.30 & $<0.0001$ \\
\hline Prosthetic heart valve & 4,805 & 1.38 & 762 & 1.09 & 699 & 0.86 & 2,585 & 2.68 & 622 & 0.75 & 137 & 0.75 & $<0.0001$ \\
\hline Carotid stenosis & 13,234 & 3.80 & 2434 & 3.49 & 5,395 & 6.64 & 2,989 & 3.10 & 1,965 & 2.38 & 451 & 2.46 & $<0.0001$ \\
\hline Current smoker & 65,934 & 18.92 & 14,181 & 20.31 & 18,124 & 22.29 & 12,843 & 13.31 & 16,782 & 20.35 & 4004 & 21.85 & $<0.0001$ \\
\hline Medical history panel missing & 253 & 0.07 & 32 & 0.05 & 88 & 0.11 & 67 & 0.07 & 59 & 0.07 & 7 & 0.04 & $<0.0001$ \\
\hline
\end{tabular}

Discharge antithrombotic therapy

\begin{tabular}{|c|c|c|c|c|c|c|c|c|c|c|c|c|c|}
\hline Antiplatelet & & & & & & & & & & & & & \\
\hline Yes & 266,288 & 76.36 & 57,993 & 83.02 & 63,280 & 77.75 & 8,627 & 60.70 & 73,171 & 88.65 & 13,217 & 72.11 & $<0.0001$ \\
\hline No & 38,179 & 10.95 & 4,600 & 6.58 & 5,076 & 6.24 & 22,060 & 22.84 & 3,987 & 4.83 & 2,456 & 13.40 & \\
\hline Missing & 44,248 & 12.69 & 7264 & 10.40 & 13,038 & 16.02 & 15,905 & 16.47 & 5,384 & 6.52 & 2,657 & 14.50 & \\
\hline \multicolumn{14}{|l|}{ Anticoagulant } \\
\hline Yes & 84,848 & 24.33 & 10,986 & 15.73 & 13,547 & 16.64 & 44,950 & 46.54 & 10,866 & 13.16 & 4,499 & 24.54 & $<0.0001$ \\
\hline No & 219,619 & 62.98 & 51,607 & 73.88 & 54,809 & 67.34 & 35,737 & 37.00 & 66,292 & 80.31 & 11,174 & 60.96 & \\
\hline Missing & 44,248 & 12.69 & 7,264 & 10.40 & 13,038 & 16.02 & 15,905 & 16.47 & 5,384 & 6.52 & 2,657 & 14.50 & \\
\hline \multicolumn{14}{|c|}{ Hospital characteristics } \\
\hline \multicolumn{14}{|c|}{$\begin{array}{l}\text { Annual ischemic stroke } \\
\text { admissions }\end{array}$} \\
\hline $\mathrm{N}$, median & 348,695 & 248.50 & 69,850 & 244.25 & 81,387 & 243.92 & 96,587 & 270.92 & 82,541 & 230.11 & 18,330 & 263.54 & $<0.0001$ \\
\hline
\end{tabular}


Table 1 Baseline and discharge characteristics in the primary analysis cohort with documented IS subtype $(n=348,715)$ (continued)

\begin{tabular}{|c|c|c|c|c|c|c|c|c|c|c|c|c|c|}
\hline \multirow{2}{*}{$\begin{array}{r}\text { Variable } \\
25 \text { th }\end{array}$} & \multicolumn{2}{|c|}{$\begin{array}{l}\text { Overall } \\
(n=348,715)\end{array}$} & \multicolumn{2}{|c|}{$\begin{array}{l}\text { CS } \\
(n=69,857)\end{array}$} & \multicolumn{2}{|c|}{$\begin{array}{l}\text { LAA } \\
(n=81,394)\end{array}$} & \multicolumn{2}{|c|}{$\begin{array}{l}\text { CE } \\
(n=96,592)\end{array}$} & \multicolumn{2}{|c|}{$\begin{array}{l}\text { SVO } \\
(\mathrm{n}=82,542)\end{array}$} & \multicolumn{2}{|c|}{$\begin{array}{l}\text { Stroke of } \\
\text { other } \\
\text { determined } \\
\text { etiology } \\
(n=18,330)\end{array}$} & \multirow[t]{2}{*}{$p$} \\
\hline & & 165.68 & & 166.52 & & 163.74 & & 178.85 & & 156.15 & & 169.57 & \\
\hline 75th & & 391.08 & & 397.85 & & 373.71 & & 414.49 & & 353.63 & & 418.06 & \\
\hline Missing (\%) & & 0.01 & & 0.01 & & 0.01 & & 0.01 & & 0.00 & & 0.00 & \\
\hline \multicolumn{14}{|c|}{ Number of beds } \\
\hline $\mathrm{N}$, median & 344,724 & 393 & 69,185 & 396 & 80,382 & 391 & 95,452 & 417 & 81,566 & 357 & 181,39 & 440 & $<0.0001$ \\
\hline 25th & & 253 & & 259 & & 251 & & 267 & & 230 & & 258 & \\
\hline 75th & & 625 & & 650 & & 599 & & 657 & & 546 & & 672 & \\
\hline Missing (\%) & & 1.14 & & 0.96 & & 1.24 & & 1.18 & & 1.18 & & 1.04 & \\
\hline \multicolumn{14}{|l|}{ Region } \\
\hline West & 66,748 & 19.14 & 12,067 & 17.27 & 15,236 & 18.72 & 19,155 & 19.83 & 16,271 & 19.71 & 4,019 & 21.93 & $<0.0001$ \\
\hline South & 140,617 & 40.32 & 28,194 & 40.36 & 33,520 & 41.18 & 37,336 & 38.65 & 34,477 & 41.77 & 7,090 & 38.68 & \\
\hline Midwest & 69,700 & 19.99 & 14,750 & 21.11 & 16,861 & 20.72 & 18,545 & 19.20 & 15,898 & 19.26 & 3,646 & 19.89 & \\
\hline Northeast & 71,650 & 20.55 & 14,846 & 21.25 & 15,777 & 19.38 & 21,556 & 22.32 & 15,896 & 19.26 & 3,575 & 19.50 & \\
\hline \multicolumn{14}{|c|}{ Teaching hospital } \\
\hline Yes & 272,887 & 78.26 & 54,602 & 78.16 & 63,398 & 77.89 & 77,556 & 80.29 & 62,522 & 75.75 & 14,809 & 80.79 & $<0.0001$ \\
\hline No & 71,112 & 20.39 & 14,558 & 20.84 & 17,120 & 21.03 & 17,840 & 18.47 & 18,420 & 22.32 & 3,174 & 17.32 & \\
\hline Missing & 4,716 & 1.35 & 697 & 1.00 & 876 & 1.08 & 1,196 & 1.24 & 1,600 & 1.94 & 347 & 1.89 & \\
\hline \multicolumn{14}{|l|}{ Rural location } \\
\hline Yes & 11,764 & 3.37 & 2,500 & 3.58 & 3,228 & 3.97 & 2,696 & 2.79 & 2,891 & 3.50 & 449 & 2.45 & $<0.0001$ \\
\hline No & 336,786 & 96.58 & 6,7294 & 96.33 & 78,116 & 95.97 & 93,874 & 97.19 & 79,628 & 96.47 & 17,874 & 97.51 & \\
\hline Missing & 165 & 0.05 & 63 & 0.09 & 50 & 0.06 & 22 & 0.02 & 23 & 0.03 & 7 & 0.04 & \\
\hline
\end{tabular}

Abbreviations: $\mathrm{CE}=$ cardioembolic; $\mathrm{CS}=$ cryptogenic stroke; IS = ischemic stroke; $\mathrm{LAA}=$ large artery atherosclerosis; $\mathrm{MI}=$ myocardial infarction; $\mathrm{ND}=$ not documented; OTH = other determined etiology; PVD = peripheral vascular disease; SVO = small vessel occlusion; UTD = undetermined.

with a history of AF. A $p$ value $<0.05$ was significant in final models.

\section{Data availability}

Data were collected by the American Heart Association (the steward of the data according to contracts between the American Heart Association and participating hospitals) and are stored securely at the Duke Clinical Research Institute (DCRI). Given that data were collected for clinical care and quality improvement, rather than primarily for research, data sharing agreements require an application process for other researchers to access the data. Interested researchers can submit proposals to utilize GWTG for research purposes, including for validation purposes. Proposals can be submitted at www.heart.org/qualityresearch. Additional information regarding the statistical analysis plan and analytic code may also be available from DCRI upon request.

\section{Results}

Among 827,949 patients from 1,936 sites, 348,175 (42.1\%) had IS with documented subtype (see the figure for flowchart). Those included, compared with excluded, were more likely to be interfacility transfers and treated at higher volume and academic centers and more likely to have complete medical history data and higher rates on defect-free care (achievement and qualities measures); however, they also had higher LOS and reduced rates of discharge home and independence at discharge (table e-1, links.lww.com/CPJ/A135).

Overall, 69,857 (20.0\%) of the 348,175 included patients with documented etiology were classified as CS, $81,394(23.3 \%)$ as LAA, 96,592 (27.7\%) as CE, 82,542 (23.7\%) as SVD, and 18,330 (5.3\%) as OTH. Among patients with CS, $51.0 \%$ were undetermined, $19.9 \%$ were unspecified, and $28.7 \%$ were 
multiple etiologies. On univariate analysis comparing across groups (table 1), patients with CS were slightly younger, less often white, more often privately insured, less often arrived by EMS, and had lower NIHSS scores than patients with CE. Patients with CS were also more likely to be admitted to smaller bed size and volume hospitals than patients with CE. In-hospital care and treatments differed by stroke subtype such that patients with CS achieve similar rates on quality and achievement measures as other subtypes except that tPA treatment was lower in those with OTH subtype. In-hospital mortality and LOS were lower in patients with CS compared with patients with CE or LAA but higher than those with SVD. Patients with CS were more likely to be ambulating at discharge and be discharged home than patients with CE or LAA (table e2, links.lww.com/CPJ/A135).

In multivariable analysis for the outcomes of interest (table 2 and table e-3, links.lww.com/CPJ/A135), patients with CS were more likely to receive tPA within 4.5 hours of last known well than all other groups but less likely to receive early antithrombotic therapy than other subtypes except OTH. Patients with CS had lower mortality and LOS than CE, LAA, and OTH subtypes but higher than SVO. Discharge home was more likely in patients with CS than all other subtypes, and patients with CS were more likely to be independent $(\mathrm{mRS}<3)$ than patients with LAA, OTH, or SVO subtypes. In sensitivity analysis excluding patients with CS with either unspecified or multiple etiologies or those with a previous history of AF but for whom the current index stroke was felt to be cryptogenic, the results were similar (tables e-4 to e-6).

\section{Discussion}

In this nationwide analysis of current clinical practice, we observed that stroke subtype is documented in approximately $50 \%$ of records, raising an important opportunity for quality improvement, and among ISs with documented subtype in
GWTG-Stroke, CS accounts for one-fifth of discharges. Furthermore, we noted that patients with CS were more likely to receive thrombolysis within 4.5 hours and have lower mortality and discharge outcomes than other subtypes except for patients with small vessel disease independent of initial stroke severity.

Our results have several implications for clinical practice. First, our finding that patients with CS have different risk factors and prognosis justifies identifying these patients in routine clinical practice. However, stroke subtype classification should require standardized diagnostic evaluation and review by qualified clinicians to apply TOAST or other criteria. Second, we found that a substantial number of patients are classified as CS, and within this category, the identification of radiographic picture of suspected embolic stroke of undetermined source (ESUS) should probably trigger further advanced diagnostic evaluation for specific treatable causes such as occult paroxysmal atrial fibrillation $^{19,20}$ and PFO. ${ }^{5-7}$ Furthermore, new strategies to prevent recurrent stroke in patients with ESUS without AF are of major clinical interest and the subject of several recent and ongoing clinical trials. ${ }^{21,22}$ A more complete understanding of CS through comprehensive registry-based collection could provide valuable new insights into which subjects may be at a greater risk of recurrent stroke.

These implications suggest an opportunity for ongoing quality improvement in stroke subtype documentation. Future initiatives could measure, track, and intervene to improve rates of subtype classification and assess whether documentation is linked to increased use of established therapies such as anticoagulation for atrial fibrillation-related stroke and interventions including carotid revascularization for symptomatic stenosis and PFO closure for younger patients. Improved subtyping would also lead to better provision of proven secondary prevention strategies in patients with known etiologies, advanced diagnostic evaluation such as long-term atrial fibrillation monitoring in patients with ESUS, and identification of patients for clinical trials.

Table 2 Absolute rates and adjusted odds ratios and 95\% Cls for discharge outcomes by stroke subtype

\begin{tabular}{|c|c|c|c|c|c|c|c|c|}
\hline & \multicolumn{2}{|c|}{ In-hospital mortality } & \multicolumn{2}{|c|}{ Independent ambulation at discharge } & \multicolumn{2}{|c|}{ Discharge home } & \multicolumn{2}{|c|}{ mRS score $0-2$ at discharge } \\
\hline & $\%$ & OR $(95 \% \mathrm{Cl})$ & $\%$ & OR $(95 \% \mathrm{Cl})$ & $\%$ & OR $(95 \% \mathrm{Cl})$ & $\%$ & OR $(95 \% \mathrm{Cl})$ \\
\hline LAA & 5.91 & $1.46(1.36-1.57)$ & 45.21 & $0.88(0.83-0.94)$ & 45.17 & $0.78(0.76-0.81)$ & 36.03 & $0.88(0.81-0.95)$ \\
\hline CE & 5.68 & $1.14(1.07-1.21)$ & 43.85 & $1.01(0.96-1.07)$ & 44.00 & $0.93(0.90-0.96)$ & 35.08 & $1.05(0.99-1.12)$ \\
\hline svo & 1.18 & $0.62(0.56-0.69)$ & 56.25 & $0.91(0.86-0.97)$ & 56.41 & $0.88(0.85-0.91)$ & 48.07 & $0.94(0.88-1.01)$ \\
\hline Отн & 4.12 & $1.47(1.33-1.63)$ & 54.64 & $0.86(0.77-0.96)$ & 54.76 & $0.75(0.71-0.79)$ & 44.92 & $0.80(0.73-0.87)$ \\
\hline CS (ref) & 2.86 & - & 53.89 & - & 55.32 & - & 44.98 & - \\
\hline
\end{tabular}

Abbreviations: CE = cardioembolic; CI = confidence interval; CS = cryptogenic stroke; LAA = large artery atherosclerosis; mRS = modified Rankin Scale; SVO = small vessel occlusion; OR = odds ratio; OTH = other determined cause.

a Models were adjusted for age, sex, race, atrial fibrillation, prosthetic heart valve, previous stroke or transient ischemic attack, coronary artery disease or previous myocardial infarction, carotid stenosis, peripheral vascular disease, hypertension, dyslipidemia, smoking, arrival during off hours (vs. regular hours), emergency medical services arrival, annual ischemic stroke admission, bed size, region, hospital type (academic vs not), urban/rural location, and NIHSS scores. 
Compared with other population-based and hospital-based registries of IS in the United States, ${ }^{12,14,15,17,23}$ the rate of CS in our study is lower (20\% vs $36-41 \%)$. Many of the previous studies were published before modern imaging including routine use of brain MRI and vascular imaging such as $\mathrm{CT}$ and magnetic resonance angiography. It is also possible that when multiple etiologies were identified, clinicians chose to select the most likely subtype, a practice that would not have been allowed in research studies where strict adherence to the TOAST subtype classification schema would have been followed. Unlike other studies, we did not find that patients with CS were younger than patients without CS, disproportionately affected minorities, or had differences in medical risk factors with the exception of less cardiac disease history. ${ }^{15,16,24}$

We observed that patients with CS had milder initial stroke severity than CE stroke and LAA subtypes. Despite this, patients with CS were more likely to receive thrombolytic therapy within 4.5 hours of last known well than all other subtypes independent of initial stroke severity. Patients with CS were more likely to have better outcomes than all subtypes except those with small vessel disease. Unmeasured confounding factors including infarct volume and some poststroke complications, not available or analyzed in this study, may explain these findings.

Although simple, the TOAST classification system has only modest interrater reliability. ${ }^{25,26}$ Compared with the TOAST system, the Causative Classification of Stroke System (CCS, available at ccs.mgh.harvard.edu) ${ }^{27}$ and Atherosclerosis, Small-vessel disease, Cardioembolism and Other causes methods ${ }^{28}$ have higher interrater reliability, but are more complex, require richly detailed clinical, laboratory and imaging data, and are, therefore, less commonly used in practice or in research. ${ }^{29,30}$ A previous study also noted that the TOAST classification system and the CCS, which uses a web-based semiautomated approach, have excellent agreement and that only $5.8 \%$ of patients with CS by TOAST would have been reclassified to a definite subtype by CCS. ${ }^{31}$

Our study has other limitations. First, selection bias may have affected our results because stroke subtype documentation was more common at larger, teaching hospitals and associated with better quality and achievement measure performance, suggesting that our results may disproportionately reflect practices from high-performance, large, academic centers and thereby limit the generalizability of our findings. Second, as noted above, there is a potential for misclassification error, given the modest interrater reliability of the TOAST classification system and its use outside the research setting. Because the expertise and training of clinicians involved in the determination of stroke subtype is not known, we cannot verify that a standard approach to diagnostic testing and subtype adjudication was used between hospitals or even within hospitals. Third, we are unable to
Given the implications of CS on diagnostic evaluation and

management, optimizing documentation of ischemic stroke subtype at all levels of hospitals should be a major public health priority.

evaluate the details of the diagnostic tests completed and whether results conformed with TOAST categories (e.g., LAA requires $>50 \%$ ipsilateral stenosis). We are also unable to verify whether infarct patterns met established criteria for either small vessel disease (infarct size $<1.5 \mathrm{~cm}$ in maximum diameter), or ESUS, or CE stroke (cortical or multi-infarct pattern). Thus, we cannot independently validate the TOAST subtype assignment at GWTG-Stroke sites. Fourth, it is possible that some stroke mimics were disproportionately misclassified as CS. Finally, residual or unmeasured confounding may explain some of our findings.

In a large contemporary nationwide analysis, we observed that CS accounts for approximately one-fifth of IS subtypes. Furthermore, we confirm that outcomes in this group are generally intermediate between those of CE and large artery atherosclerotic stroke and small vessel disease. Given the implications of CS on diagnostic evaluation and management, optimizing documentation of IS subtype at all levels of hospitals should be a major public health priority.

\section{Study funding}

This study was funded by the American Heart Association/ American Stroke Association.

\section{Disclosure}

S. Prabhakaran. reports that he is a member of the AHA/ ASA Cryptogenic Stroke Oversight Committee. S.R. Messe and D. Kleindorfer report no disclosures. E.E. Smith discloses that he is a member of the GWTG steering committee. G.C. Fonarow reports serving on the GWTG steering committee; receiving grant funding from the Patient-Centered Outcome Research Institute; and being an employee of the University of California, which has a patent on an endovascular therapy. H. Xu, X. Zhao, B. Lytle, and J. Cigarroa report no disclosures. L.H. Schwamm reports serving as chair of the American Heart Association/ American Stroke Association GWTG stroke clinical work group and hospital accreditation science committee; 
TAKE-HOME POINTS

$\rightarrow$ Among records with documented subtype in a nationwide registry, CS accounts for $20 \%$ of IS.

$\rightarrow$ Such patients experience an intermediate discharge outcome better than CE and LAA subtypes but worse than SVO.

$\rightarrow$ Improved subtype documentation and further research into CS are warranted to improve care and outcomes for patients with stroke.

serving as a stroke systems consultant to the Massachusetts Department of Public Health; and serving as a scientific consultant regarding trial design and conduct to Penumbra (data and safety monitoring committee, Separator 3D and MIND trial) and Medtronic (Victory AF and Stroke AF trials). Full disclosure form information provided by the authors is available with the full text of this article at Neurology.org/cp.

\section{Publication history}

Received by Neurology: Clinical Practice March 19, 2019. Accepted in final form August 21, 2019.

\section{Appendix Authors}

\begin{tabular}{lll}
\hline Name & Location & Contribution \\
\hline $\begin{array}{l}\text { Shyam } \\
\text { Mrabhakaran, MS }\end{array}$ & $\begin{array}{l}\text { University of Chicago, } \\
\text { Illinois }\end{array}$ & $\begin{array}{l}\text { Drafting/revising the } \\
\text { manuscript; study concept } \\
\text { or design; and analysis or } \\
\text { interpretation of the data }\end{array}$ \\
\hline $\begin{array}{l}\text { Steven R. Messé, } \\
\text { MD }\end{array}$ & $\begin{array}{l}\text { University of } \\
\text { Pennsylvania, } \\
\text { Philadelphia, PA }\end{array}$ & $\begin{array}{l}\text { Drafting/revising the } \\
\text { manuscript }\end{array}$ \\
\hline $\begin{array}{l}\text { Dawn } \\
\text { Kleindorfer, MD }\end{array}$ & $\begin{array}{l}\text { University of Cincinnati, } \\
\text { Cincinnati, OH }\end{array}$ & $\begin{array}{l}\text { Drafting/revising the } \\
\text { manuscript; study concept } \\
\text { or design; and analysis or } \\
\text { interpretation of the data }\end{array}$ \\
\hline $\begin{array}{l}\text { Eric E. Smith, MD, University of Calgary, } \\
\text { MPH }\end{array}$ & $\begin{array}{l}\text { Study concept or design; } \\
\text { and analysis or } \\
\text { interpretation of the data }\end{array}$ \\
\hline $\begin{array}{l}\text { Gregg C. } \\
\text { Fonarow, MD }\end{array}$ & UCLA, Los Angeles, CA & $\begin{array}{l}\text { Drafting/revising the } \\
\text { manuscript; data } \\
\text { acquisition; and analysis } \\
\text { or interpretation of the } \\
\text { data }\end{array}$ \\
\hline Xin Zhao, PhD & $\begin{array}{l}\text { Duke Clinical Research } \\
\text { Institute, Durham, NC }\end{array}$ & $\begin{array}{l}\text { Analysis or interpretation } \\
\text { of the data; and statistical } \\
\text { analysis }\end{array}$ \\
\hline Haolin Xu, MS & $\begin{array}{l}\text { Duke Clinical Research } \\
\text { Institute, Durham, NC }\end{array}$ & $\begin{array}{l}\text { Study concept or design; } \\
\text { analysis or interpretation } \\
\text { of the data; and statistical } \\
\text { analysis }\end{array}$ \\
\hline & & \\
\hline
\end{tabular}

Appendix (continued)

\begin{tabular}{lll}
\hline Name & Location & Contribution \\
\hline $\begin{array}{l}\text { Barbara Lytle, } \\
\text { PhD }\end{array}$ & $\begin{array}{l}\text { Duke Clinical Research } \\
\text { Institute, Durham, NC }\end{array}$ & $\begin{array}{l}\text { Analysis or interpretation } \\
\text { of the data; and study } \\
\text { supervision }\end{array}$ \\
\hline $\begin{array}{l}\text { Joaquin } \\
\text { Cigarroa, MD }\end{array}$ & $\begin{array}{l}\text { Oregon Health and } \\
\text { Sciences University, } \\
\text { Portland, OR }\end{array}$ & $\begin{array}{l}\text { Drafting/revising the } \\
\text { manuscript; and analysis } \\
\text { or interpretation of data }\end{array}$ \\
\hline $\begin{array}{l}\text { Lee H. } \\
\text { Schwamm, MD }\end{array}$ & $\begin{array}{l}\text { Massachusetts General } \\
\text { Hospital, Boston, MA }\end{array}$ & $\begin{array}{l}\text { Drafting/revising the } \\
\text { manuscript; and study } \\
\text { concept or design }\end{array}$ \\
\hline
\end{tabular}

\section{References}

1. Benjamin EJ, Blaha MJ, Chiuve SE, et al. Heart disease and stroke statistics-2017 update: a report from the American Heart Association. Circulation 2017;135: e146-e603.

2. Adams HP Jr, Bendixen BH, Kappelle LJ, et al. Classification of subtype of acute ischemic stroke. Definitions for use in a multicenter clinical trial. TOAST. Trial of ORG 10172 in Acute Stroke Treatment. Stroke 1993;24:35-41.

3. Yaghi S, Kamel H, Elkind MS. Potential new uses of non-vitamin $\mathrm{K}$ antagonist oral anticoagulants to treat and prevent stroke. Neurology 2015;85:1078-1084.

4. Diener HC, Bernstein R, Hart R. Secondary stroke prevention in cryptogenic stroke and embolic stroke of undetermined source (ESUS). Curr Neurol Neurosci Rep 2017;17:64.

5. Mas JL, Derumeaux G, Guillon B, et al. Patent foramen ovale closure or anticoagulation vs. antiplatelets after stroke. N Engl J Med 2017;377:1011-1021.

6. Saver JL, Carroll JD, Thaler DE, et al. Long-term outcomes of patent foramen ovale closure or medical therapy after stroke. N Engl J Med 2017;377:1022-1032.

7. Sondergaard L, Kasner SE, Rhodes JF, et al. Patent foramen ovale closure or antiplatelet therapy for cryptogenic stroke. N Engl J Med 2017;377:1033-1042.

8. Grau AJ, Weimar C, Buggle F, et al. Risk factors, outcome, and treatment in subtypes of ischemic stroke: the German stroke data bank. Stroke 2001;32:2559-2566.

9. Kolominsky-Rabas PL, Weber M, Gefeller O, Neundoerfer B, Heuschmann PU. Epidemiology of ischemic stroke subtypes according to TOAST criteria: incidence, recurrence, and long-term survival in ischemic stroke subtypes: a population-based study. Stroke 2001;32:2735-2740.

10. Lee BI, Nam HS, Heo JH, Kim DI. Yonsei Stroke Registry. Analysis of 1,000 patients with acute cerebral infarctions. Cerebrovasc Dis 2001;12:145-151.

11. Li L, Yiin GS, Geraghty OC, et al. Incidence, outcome, risk factors, and long-term prognosis of cryptogenic transient ischaemic attack and ischaemic stroke: a population-based study. Lancet Neurol 2015;14:903-913.

12. Petty GW, Brown RD Jr, Whisnant JP, Sicks JD, O'Fallon WM, Wiebers DO Ischemic stroke subtypes: a population-based study of incidence and risk factors. Stroke 1999;30:2513-2516.

13. Putaala J, Metso AJ, Metso TM, et al. Analysis of 1008 consecutive patients aged 15 to 49 with first-ever ischemic stroke: the Helsinki young stroke registry. Stroke 2009;40: 1195-1203.

14. Sacco RL, Ellenberg JH, Mohr JP, et al. Infarcts of undetermined cause: the NINCDS Stroke Data Bank. Ann Neurol 1989;25:382-390.

15. Schneider AT, Kissela B, Woo D, et al. Ischemic stroke subtypes: a population-based study of incidence rates among blacks and whites. Stroke 2004;35:1552-1556.

16. Schulz UG, Rothwell PM. Differences in vascular risk factors between etiological subtypes of ischemic stroke: importance of population-based studies. Stroke 2003;34 2050-2059.

17. White $\mathrm{H}$, Boden-Albala $\mathrm{B}$, Wang $\mathrm{C}$, et al. Ischemic stroke subtype incidence among whites, blacks, and Hispanics: the Northern Manhattan Study. Circulation 2005;111: 1327-1331.

18. Ormseth $\mathrm{CH}$, Sheth KN, Saver JL, Fonarow GC, Schwamm LH. The American Heart Association's Get With the Guidelines (GWTG)-Stroke development and impact on stroke care. Stroke Vasc Neurol 2017;2:94-105.

19. Gladstone DJ, Spring M, Dorian P, et al. Atrial fibrillation in patients with cryptogenic stroke. N Engl J Med 2014;370:2467-2477.

20. Sanna T, Diener HC, Passman RS, et al. Cryptogenic stroke and underlying atrial fibrillation. N Engl J Med 2014;370:2478-2486.

21. Yaghi S, Kamel H, Elkind MSV. Atrial cardiopathy: a mechanism of cryptogenic stroke. Expert Rev Cardiovasc Ther 2017;15:591-599.

22. Hart RG, Sharma M, Mundl H, et al. Rivaroxaban for stroke prevention after embolic stroke of undetermined source. N Engl J Med 2018;378:2191-2201.

23. Woo D, Gebel J, Miller R, et al. Incidence rates of first-ever ischemic stroke subtypes among blacks: a population-based study. Stroke 1999;30:2517-2522.

24. Jacobs BS, Boden-Albala B, Lin IF, Sacco RL. Stroke in the young in the northern Manhattan stroke study. Stroke 2002;33:2789-2793.

25. Meschia JF, Barrett KM, Chukwudelunzu F, et al. Interobserver agreement in the Trial of ORG 10172 in Acute Stroke Treatment classification of stroke based on retrospective medical record review. J Stroke Cerebrovasc Dis 2006;15:266-272.

26. Goldstein LB, Jones MR, Matchar DB, et al. Improving the reliability of stroke subgroup classification using the Trial of ORG 10172 in Acute Stroke Treatment (TOAST) criteria. Stroke 2001;32:1091-1098. 
27. Ay H, Benner $\mathrm{T}$, Arsava EM, et al. A computerized algorithm for etiologic classification of ischemic stroke: the causative classification of stroke system. Stroke 2007;38:2979-2984.

28. Amarenco P, Bogousslavsky J, Caplan LR, Donnan GA, Wolf ME, Hennerici MG. The ASCOD phenotyping of ischemic stroke (Updated ASCO Phenotyping). Cerebrovasc Dis 2013;36:1-5.

29. Gökçal E, Niftaliyev E, Asil T. Etiological classification of ischemic stroke in young patients: a comparative study of TOAST, CCS, and ASCO. Acta Neurol Belg 2017;117:643-648.
30. Marnane M, Duggan CA, Sheehan OC, et al. Stroke subtype classification to mechanism-specific and undetermined categories by TOAST, A-S-C-O, and causative classification system: direct comparison in the North Dublin population stroke study. Stroke 2010;41:1579-1586.

31. Lanfranconi S, Markus HS. Stroke subtyping for genetic association studies? A comparison of the CCS and TOAST classifications. Int J Stroke 2013;8: 626-631.

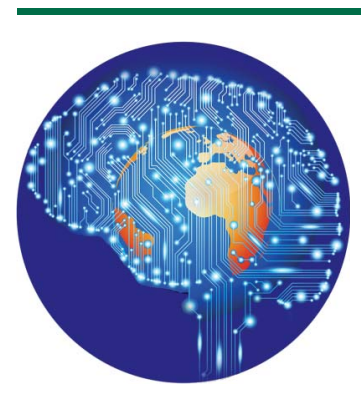

\section{Practice Current: An interactive exchange on} controversial topics

Share your own best practices.

Read commentary with expert opinion.

Explore results on an interactive world map. 